\title{
Removal of False Blood Vessels Using Shape Based Features and Image Inpainting
}

\author{
Amna Waheed, Zahra Waheed, M. Usman Akram, and Arslan Shaukat \\ Department of Computer Engineering, National University of Sciences \& Technology, Islamabad 44000, Pakistan \\ Correspondence should be addressed to M. Usman Akram; usman.akram@ceme.nust.edu.pk
}

Received 25 March 2015; Accepted 17 May 2015

Academic Editor: Kourosh Kalantar-Zadeh

Copyright (C) 2015 Amna Waheed et al. This is an open access article distributed under the Creative Commons Attribution License, which permits unrestricted use, distribution, and reproduction in any medium, provided the original work is properly cited.

\begin{abstract}
Automated quantification of blood vessels in human retina is the fundamental step in designing any computer-aided diagnosis system for ophthalmic disorders. Detection and analysis of variations in blood vessels can be used to diagnose several ocular diseases like diabetic retinopathy. Diabetic Retinopathy is a progressive vascular disorder caused due to variations in blood vessels of retina. These variations bring different abnormalities like lesions, exudates, and hemorrhages in human retina which make the vessel detection problematic. Therefore, automated retinal analysis is required to cater the effect of lesions while segmenting blood vessels. The proposed framework presents two improved approaches to carry out vessel segmentation in the presence of lesions. The paper mainly aims to extract true vessels by reducing the effect of abnormal structures significantly. First method is a supervised approach which extracts true vessels by performing region based analysis of retinal image, while second method intends to remove lesions before extracting blood vessels by using an inpainting technique. Both methods are evaluated on STARE and DRIVE and on our own database AFIO. Experimental results demonstrate the excellence of the proposed system.
\end{abstract}

\section{Introduction}

The most common disease that occurs in human retina is Diabetic Retinopathy (DR) which is one of the main causes of vision loss [1]. It is caused due to increased insulin level in the blood which brings different variations in vascular pattern of retina either by increasing vessel width or by fluid leakage and so forth [2]. This can cause blindness as the disease advances if not treated in time. Abnormal false vessels appear on the surface of retina as the disease progresses; therefore, it is essential to detect it timely to lower the risk of blindness. Hence, for the timely diagnosis of retinopathy, there is a need to analyze fundus images accurately. The normal features of fundus images are blood vessels, fovea, optic disk, and macula, as illustrated in Figure 1.

Blood vessel is the most stable and secure component of human retina which sets the foundation of retina based authentication system [3]. It is the most reliable biometric system due to distinctiveness of blood vessels which are unique in every individual even in identical twins. In DR images, these blood vessels undergo some abnormal changes like the appearance of lesions, exudates, and so forth along with the true vessels. Nonproliferative Diabetic Retinopathy (NPDR) and Proliferative Diabetic Retinopathy (PDR) are the two main stages of DR. NPDR is the early stage of DR which includes appearance of hemorrhages and microaneurysms due to leakage of thin vessels which later become hard exudates with the inclusion of fats and proteins [2], and all abnormal structures that appear due to Diabetic Retinopathy are shown in Figure 2. Moreover, in advance phase of PDR, these small vessels appear as white spots known as cotton wool spots when retina gets deprived of enough oxygen. Then retina signals the body to grow more new fragile vessels to maintain the adequate level of oxygen in retina; this is known as neovascularization. So PDR can be further divided into two stages, that is, NVD (neovascularization on optic disc) and NVE (neovascularization elsewhere).

Commonly used biometric systems are fingerprint, iris, face, and speech recognition [4]. Among all biometric systems, retinal recognition is the most stable system due to high 


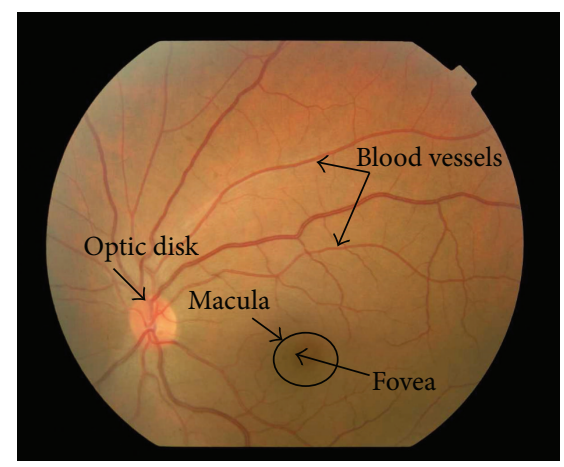

FIGURE 1: Fundus image with normal features.

stability and reliability of human retina as it lies at the back of human eye. Person's identification is performed on the basis of his unique blood vessels pattern. Appearance of different abnormal structures in blood vessels makes the detection of disease difficult. DR produces lesions spots which cause appearance of false vessels in the vascular pattern of DR image. These false vessels due to lesions appear in the form of bunches and holes along with the true vessels as indicated with red circle in Figure 3; hence, they make the blood vessel segmentation a challenging task. Development of false vessels leads to the increase of false positives which may lead to misidentification, hence degrading the performance of the overall system. So an improved system must be designed to diminish the effect of lesions in order to remove false positives. Therefore, there comes a need of developing an automated and accurate vessel extraction algorithm in the presence of lesions.

In this paper, two improved methods for the accurate segmentation of blood vessels are proposed. Both methods aim to get a segmented blood vessel with the true vessels only by removing lesions effects and false vessels. Method I is a classification based method which works on each region to classify it as true vessel or false vessel. Method II mainly aims to remove lesions effect by using inpainting technique before segmenting blood vessels. Both methods intend to get the true vessels as the output and hence remove false vessel detection significantly.

The paper is structured as follows. Section 2 discusses several previous methods on blood vessel segmentation. Section 3 describes proposed methodology in detail and results are discussed in Section 4. Section 5 finally concludes the paper.

\section{Related Work}

Various works have been carried out in the past few years on the accurate segmentation of blood vessels in fundus images. Vessel segmentation techniques are broadly divided into two categories: supervised and unsupervised.

Supervised methods classify each pixel as vessel and nonvessel on the basis of prior knowledge used. A classification based method was proposed in [5]. It used neural networks to classify each pixel as exudate or nonexudate using $\mathrm{Hu}$ moment features of size 7D. In [3], Local Binary Pattern (LBP) and gray-level cooccurrence matrix (GLCM) features are extracted to classify each pixel as vessel and nonvessel with the help of support vector machine (SVM) classifier. Shanmugam and Banu [6] presented Extreme Learning Machine (ELM) based classification method. Seven different features of gray-level and moment invariant based features were extracted to classify pixels as vessel or not. An improved classification based retinal blood vessel segmentation was proposed in [7]. Method used the powerful classifiers, that is, SVM and neural network (NN), to work together on the previously extracted feature sets. Artificial neural networks (ANN) were fused with the SVM to classify each pixel as vessel or nonvessel. Shruthi et al. [8] presented a novel algorithm for the detection of DR. Top-Hat and BottomHat operations based method and $k$-means clustering were used to detect retinal features. Later on, statistical features were extracted from detected retinal features which were used by $k$-nearest neighbors $(k \mathrm{NN})$ classifier in next phase of classification. Wang et al. gave a supervised hybrid approach that was proposed in [9]. Trainable hierarchical feature extraction was performed using Convolutional Neural Network (CNN) classifier. Then "winner takes all" approach was used to classify each pixel as vessel or nonvessel by employing Random Forests (RFs). An effective supervised blood vessel segmentation technique was given by Fraz et al. in [10]. He used ensemble classifier of boosted and bagged decision trees. 9D feature set was formed for classification, among 9 features best 4 features were chosen by performing feature analysis. One bagging and two boosting algorithms were used to apply three ensemble classifiers on DRIVE, STARE, and CHASE_DB1 databases.

Unsupervised techniques are classification free methods which do not use any prior knowledge. In [11], a novel segmentation technique based on Artificial Bee Colony model with the use of fuzzy $c$-means clustering was presented. Asad et al. [12] proposed a new segmentation approach using water flooding model which used the concept of water flooding over the land. Phase congruency and histogram clustering based segmentation technique was given by Tagore et al. [13]. In this method, fundus images were enhanced using phase congruency and enhanced images were segmented using hierarchical clustering based histogram thresholding. Connected component labeling based eight connected neighborhoods and label propagation were employed to get the final vessel map. Exudates extraction was performed by using maximum entropy based thresholding technique followed by preprocessing in [14]. An improved method using matched filtering (MF) with kittler minimum error thresholding technique was proposed by Odstrcilik et al. [15]. A simple approach for the extraction of blood vessel was presented by Saleh and Eswaran [16]. Firstly, images were preprocessed; then, multilevel thresholding was applied to get an indexed image with an optimum threshold. Final vessel map was generated by applying binarisation and postfiltering. 


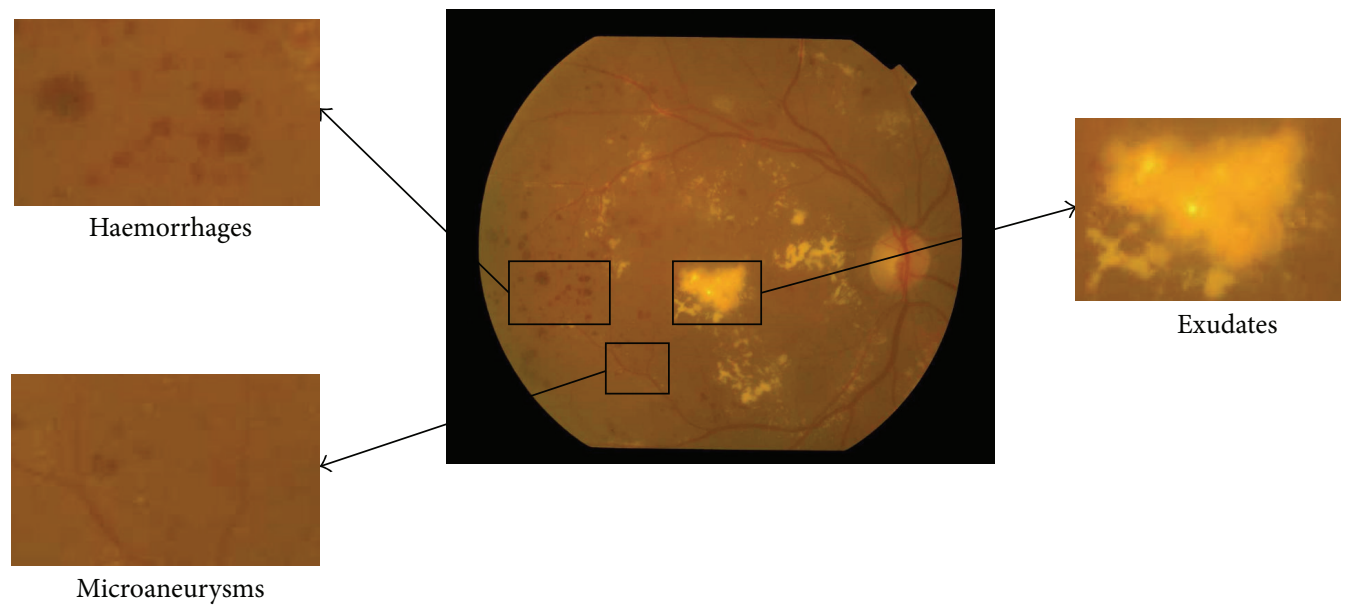

FIGURE 2: DR image with abnormal structures.

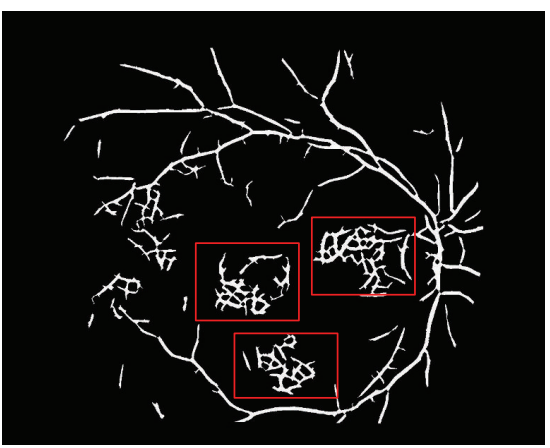

FIGURE 3: Segmented image with false vessels due to lesions.

Bhattacharjee and Chakraborty [17] presented an algorithm for accurate vessel segmentation which worked in three steps. In the first step, images were enhanced using matched filtering. In second step, local entropy thresholding was applied on enhanced images to segment the blood vessels. Lastly, connected component analysis was performed to get rid of misclassification.

It is evident from the literature that almost all previously proposed methods work better on healthy images but fail to perform well on diseased images. Occlusion of false positives in the presence of lesions is the major issue that has not been addressed effectively in the literature. Some researchers tried to cater lesions in segmentation of blood vessels but still there lies many gaps which need to be improved. These drawbacks give the motivation to develop an automated blood vessel segmentation system to suppress the effect of lesions completely in the segmented images.

\section{Proposed Method}

Blood vessel segmentation has become the most crucial task in diseased images. Many methods have been proposed previously as discussed in Section 2. Most of them fail to perform well on diseased images as they least focus on images with lesions. The effect of lesions on retinal images has been discussed extensively in first section which eventually cause occlusion of false positives in the images making the segmentation harder. This paper has addressed this problem markedly and has proposed two improved methods which aim to segment the blood vessels in the presence of lesions.

3.1. Method I. It is the supervised method whose flow diagram is shown in Figure 4. Method I first extracts vascular pattern from colored retinal images using techniques discussed in $[18,19]$. This gives a vascular pattern with both true and false structures in it. This method intends to remove false structures from true ones by doing region based analysis of blood vessels. So, this method works in three main steps: region based feature extraction, feature selection, and classification. Eventually, it gives only true vessels as an output.

3.1.1. Region Based Feature Extraction. False vessels due to lesions are developed along with true vessels in fundus images. Both false and true vessels have different morphological characteristics. False vessels appear as bunches and holes whereas true vessels are elongated with no holes and bunches as illustrated in Figure 5. So eight shape based features are extracted which best discriminate true and false vessels based on their morphology.

Therefore, different regions of fundus images are taken as candidate regions. Each candidate region is represented by a feature vector. If a retinal image $I$ has $n$ candidate regions, then set representation for an image $I$ is $I=\left\{v_{1}, v_{2}, \ldots, v_{n}\right\}$, where $v_{j}$ is a feature vector for $j$ th candidate region containing $m$ features as $v=\left\{f_{1}, f_{2}, \ldots, f_{m}\right\}$. This method formulates descriptive feature vector for each region. So, eight different shape based features are extracted and each region 


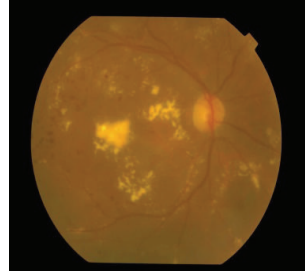

Colored retinal image

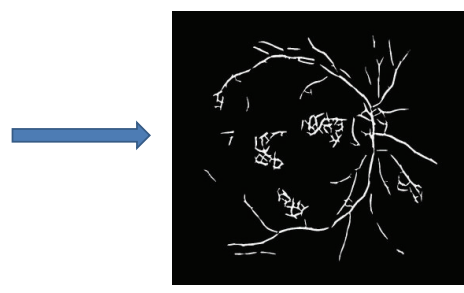

Segmented vascular pattern

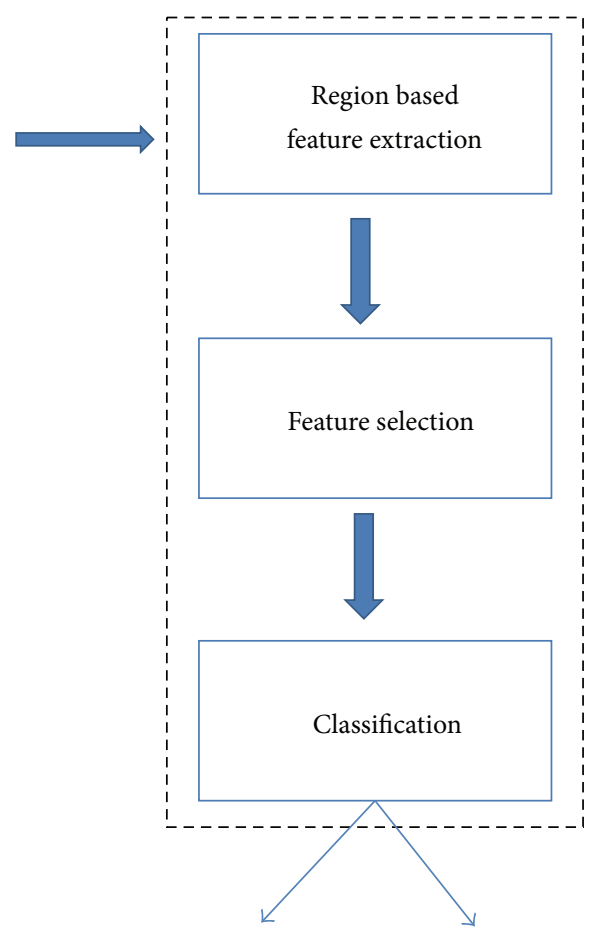

True vessel
False vessel

FIgURE 4: Flow diagram of Method I.

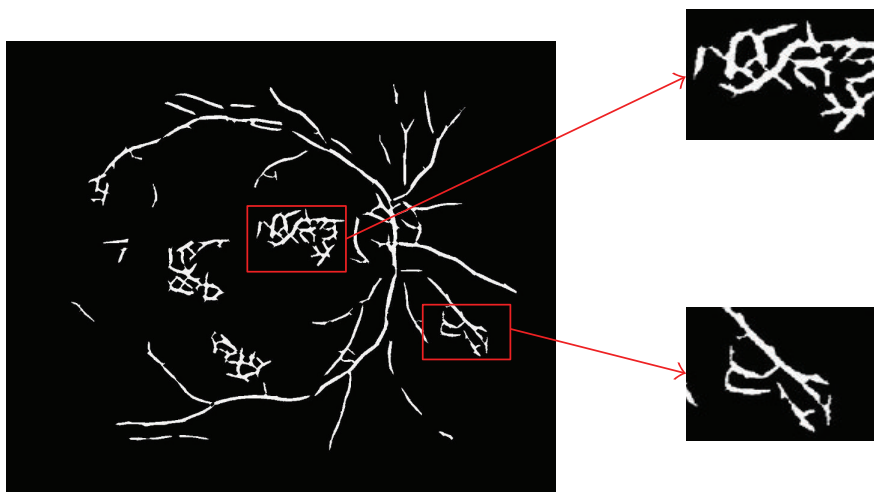

FIGURE 5: Vessel map of diseased fundus image with zoomed true and false vessels.

is represented by a feature vector of length 8 . The description of extracted features is given below.

(i) Euler number $\left(f_{1}\right)$ : it is the number of holes in the image.

(ii) Eccentricity $\left(f_{2}\right)$ : specifies circulatory of the objects in the image.

(iii) Convex area $\left(f_{3}\right)$ : it is the number of pixels in convex image.

(iv) Extent $\left(f_{4}\right)$ : it is defined as the ratio of pixels in the region to pixels in the total bounding box. (v) Filled area $\left(f_{5}\right)$ : it shows number of pixels in the filled image.

(vi) Major axis length $\left(f_{6}\right)$ : it reflects length of major axis of the ellipse.

(vii) Minor axis length $\left(f_{7}\right)$ : it specifies length of the minor axis of the ellipse.

(viii) Solidity $\left(f_{8}\right)$ : it shows proportion of pixels in the convex hull that are also in the region.

3.1.2. Feature Selection. Out of all 8 extracted features, best features are selected to support the process of classification 
using the feature selection techniques which are discussed below.

(1) Wilcoxon Rank Test. Wilcoxon rank test has been implemented in this method for selecting good features out of the extracted ones. This method ranks the key features by class separability criteria. A Wilcoxon criterion finds the absolute value of $u$-statistic of a two-sample unpaired Wilcoxon test [20].

(2) Principle Component Analysis (PCA). PCA is one of the dimensionality reduction techniques. It performs analysis of data to identify patterns and finds patterns to reduce dimensions of the dataset with the minimal loss of information [21]. It projects data into the direction with maximum variances where data variance is greater [4].

3.1.3. Classification. After selecting best features, each region of fundus image is represented by $6 \mathrm{D}$ feature vector including best discriminating features. Data distribution is analyzed carefully and reveals that SVM classifier is more suitable to be used in this method. This technique uses SVM classifier with RBF kernel for classification which requires two optimized factors: penalty factor $(C)$ and sigma $(S) .100 \times 100$ search grid is applied using 2-fold cross-validation to get optimized $S$ and $C$. Best $S$ and $C$ are selected based upon the highest accuracy. These best values are given to SVM using 3-fold cross-validation under 100 iterations to get unbiased and optimized results. It eventually classifies each region as true vessel or false vessel.

3.2. Method II. This is a classification free method which presents an inpainting based segmentation technique by neighborhood estimation. It mainly focuses on reducing the effect of lesions which appear as false positive and degrades system's performance. The overview of Method II is shown in Figure 6. This method first detects all the exudates present in diseased retinal image by using techniques discussed in [22]. It gives a binary mask of all exudates as shown in Figure 7. Method II mainly aims to reduce the effect of these exudates before segmentation by filling these exudates region using an algorithm of neighborhood based region filling (NBRF). The key idea of this method is to inpaint the lesions before blood vessel enhancement and segmentation to avoid the enhancement of artifacts upon applying enhancement and segmentation techniques.

3.2.1. Exudate Inpainting. This step takes exudate mask detected previously as an input and fills all the exudate regions in a very smooth manner by using an algorithm of neighborhood based region filling (NBRF).

(1) NBRF Algorithm. Detected exudates are inpainted using an algorithm of neighborhood based region filling (NBRF) to make image clean before enhancement and segmentation so that false positives get reduced significantly. It works in

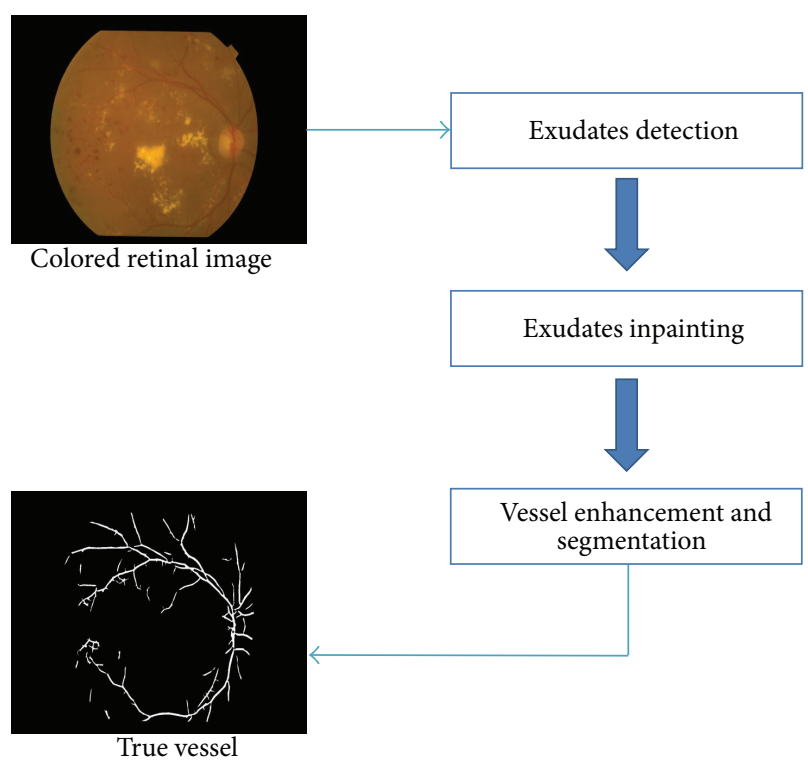

Figure 6: Flow diagram of Method II.

a radial way towards the center of the exudates. It fills exudates in a very smooth way by estimating the neighborhood and averaging the background. It runs iteratively which eventually blends all exudates within the filled region. Figure 8 shows the results of image with inpainted exudates obtained after applying NBRF algorithm.

3.2.2. Vessel Enhancement and Segmentation. After inpainting all exudates present in diseased images, vessel enhancement and segmentation are performed using techniques proposed in [5]. 2D Gabor Wavelet Transform is used for enhancement as they can be tuned to specific orientations and frequencies. Multilayered thresholding technique is used to segment the final vessel map of the image which use different thresholds iteratively. Final segmented vessel map is shown in Figure 9 which shows that by inpainting the exudates, and lesions effect has been removed and no false vessel appears in final vessel map.

\section{Experimental Results}

Performance proposed system is assessed using three databases: two publicly available databases DRIVE and STARE and our own database AFIO.

4.1. Database. Proposed system is assessed on three databases: two publicly available databases DRIVE and STARE and our own AFIO database. There are 40 fundus images in DRIVE, out of which 7 are diseased images, while all rest are healthy images [23]. Each image has a resolution of $584 \times$ 565 pixels and compressed in JPEG format. There are two sets made of the dataset: training set and testing set, each set has 20 images. STARE has total 20 images and 10 of them are diseased images [24]. Each image is of $700 \times 605$ pixels 


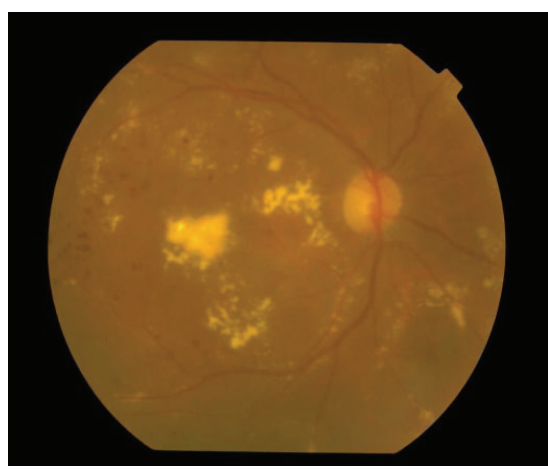

(a)

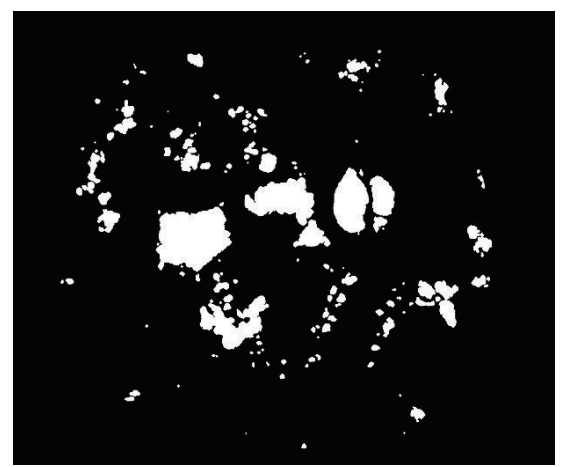

(b)

FIGURE 7: (a) Diseased retinal image, (b) exudate mask of image (a).

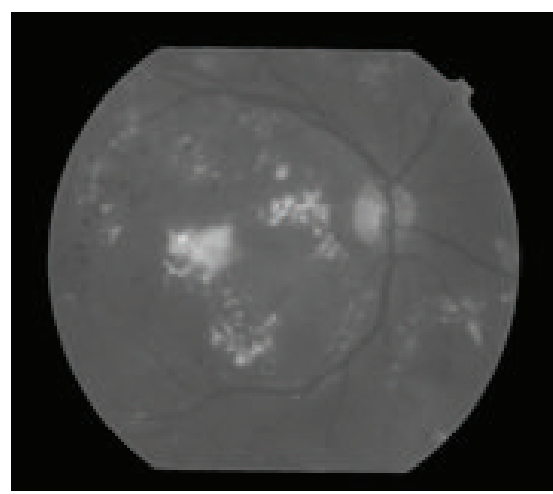

(a)

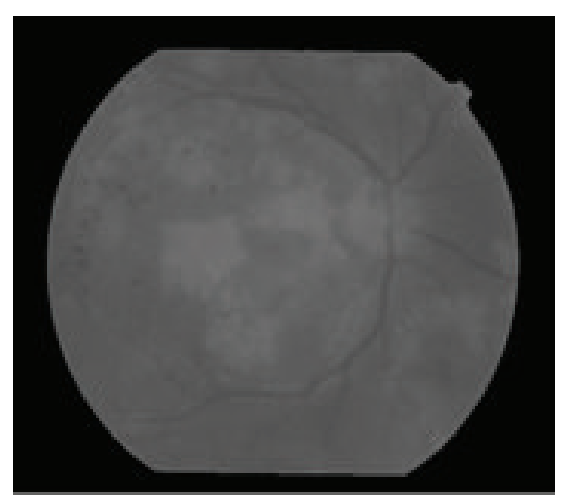

(b)

FIGURE 8: (a) Diseased retinal image, (b) exudate mask of image (a).

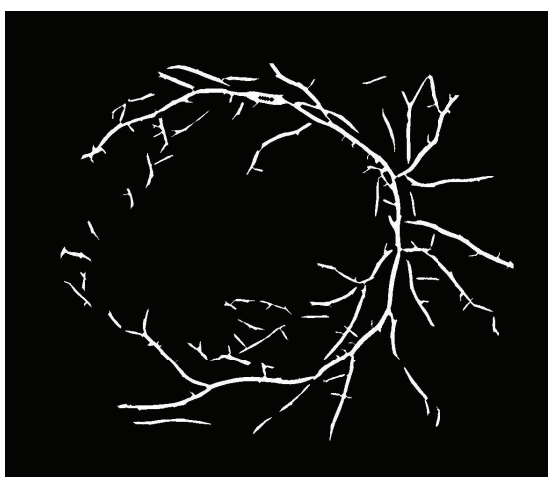

FIGURE 9: Segmented vessel map.

resolution and stored in PPM format. AFIO dataset consists of 462 total images including both healthy and unhealthy images. $1504 \times 1000$ is the resolution of each image of the dataset. Images are captured by TopCon 50EX with 30-degree FOV. Each image is stored in JPEG format. Some of the images of all databases used in the proposed system are shown in Figure 10.
4.2. Performance Measures. The evaluation of proposed system is done using performance measures, that is, accuracy, sensitivity, and specificity, which are calculated by using

$$
\begin{aligned}
\text { Sensitivity }(\mathrm{TPR}) & =\frac{T_{P}}{T_{P}+F_{N}}, \\
\text { Specificity }(\mathrm{TNR}) & =\frac{T_{N}}{T_{N}+F_{P}}, \\
\text { Accuracy } & =\frac{T_{P}+T_{N}}{T_{P}+T_{N}+F_{N}+F_{P}},
\end{aligned}
$$

where $T_{P}$ (true positive) represents the number of true vessel regions correctly classified as true vessels. $F_{P}$ (false positive) represents the number of false vessel regions classified as true vessels. $T_{N}$ (true negative) represents the number of false vessel regions correctly classified as false vessels. $F_{N}$ (false negative) represents the number of true vessels classified as false vessels.

4.3. Results Analysis. In the proposed Method I, firstly all 8 features are evaluated using feature selection technique 

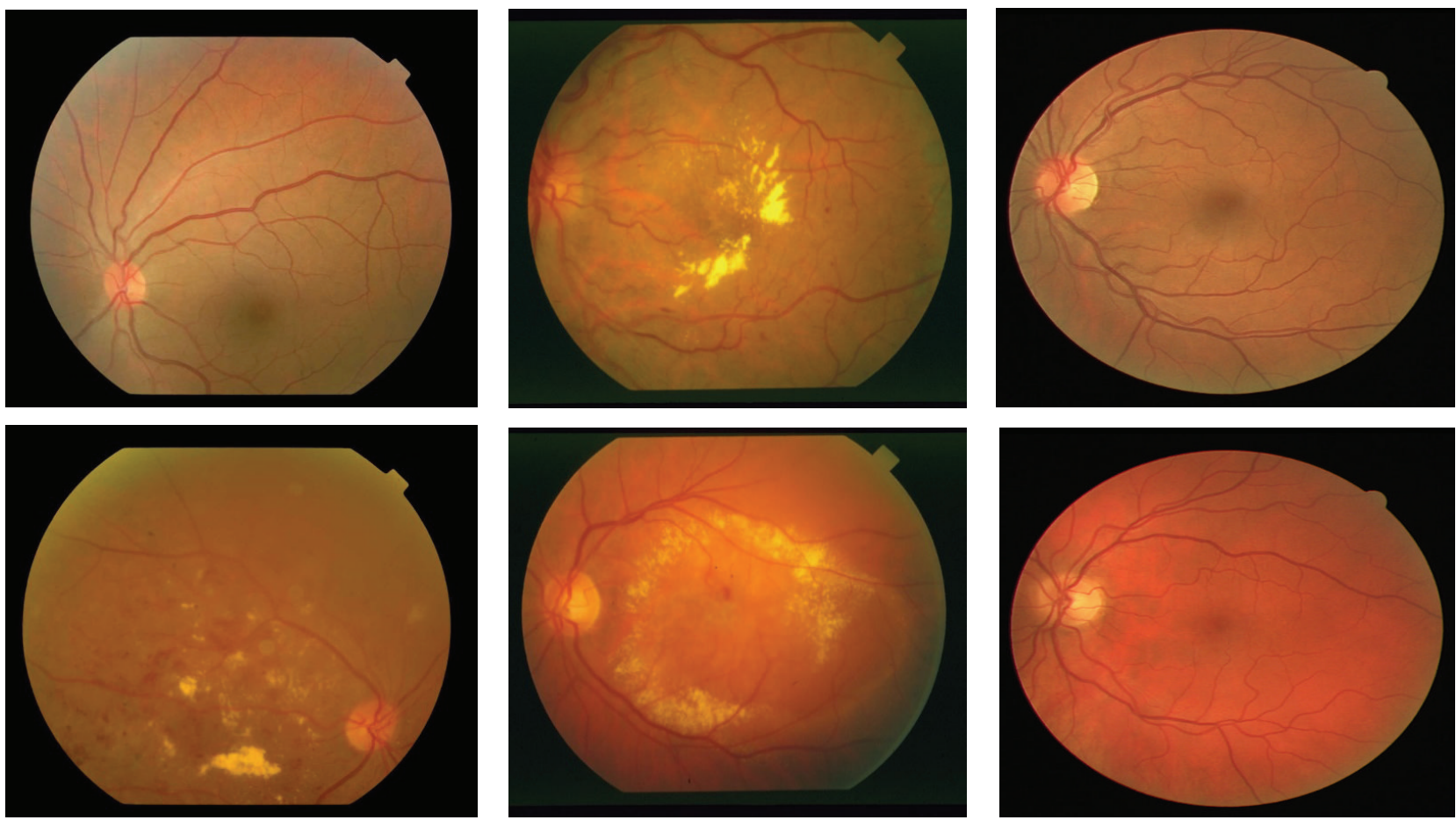

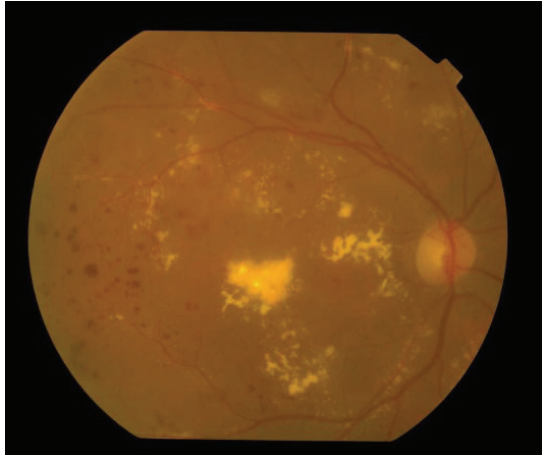

(a)

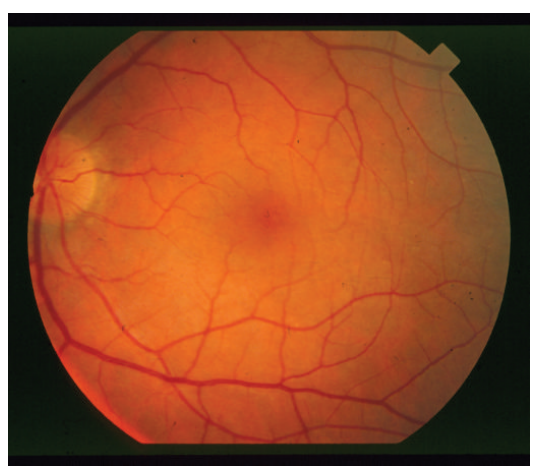

(b)

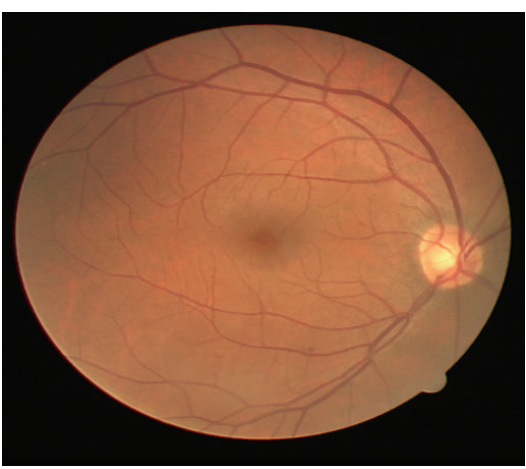

(c)

Figure 10: Databases (a) AFIO, (b) STARE, and (c) DRIVE.

Wilcoxon rank test to assess the contribution of each feature on overall system's performance. It sorts the features on the basis of their score values such that the feature with highest score value lies at the top and features with lowest value lies at the bottom. Later on, different combinations are made by combining different high ranked features, which are then given to classifier to assess the impact of features on overall accuracy of the system. Table 1 shows the performance evaluation of all features based on their score values.

4.3.1. Experimental Test Bench. Method I is evaluated on DRIVE and STARE and our own database AFIO. SVM classifier is employed with RBF kernel. Penalty factor $(C)$ and sigma $(S)$ are required, which are optimized using $100 \times$ 100 search grid under 2 -fold cross-validation. Accuracy is
TABLE 1: Performance evaluation of all features using Wilcoxon rank test.

\begin{tabular}{lccc}
\hline Feature & Name & Score & $p$ value \\
\hline$f_{2}$ & Eccentricity & $\mathbf{1 1 . 1 0 4 7}$ & $<10^{-6}$ \\
$f_{1}$ & Euler number & $\mathbf{6 . 2 3 1 0}$ & $<10^{-6}$ \\
$f_{7}$ & Minor axis length & $\mathbf{4 . 2 2 7 5}$ & $\mathbf{0 . 0 0 0 0 2 3 6}$ \\
$f_{4}$ & Extent & $\mathbf{3 . 7 3 9 7}$ & $\mathbf{0 . 0 0 0 1 8 4 2}$ \\
$f_{3}$ & Convex area & $\mathbf{2 . 2 8 2 6}$ & $\mathbf{0 . 0 2 2 5}$ \\
$f_{5}$ & Filled area & $\mathbf{2 . 1 6 5 5}$ & $\mathbf{0 . 0 3 0 4}$ \\
$f_{8}$ & Solidity & 2.0430 & 0.0411 \\
$f_{6}$ & Major axis length & 1.2908 & 0.1968 \\
\hline
\end{tabular}

calculated for all $S$ and $C$ values, each ranging from 1 to 100 . The best $C$ and $S$ are computed corresponding to highest 
TABle 2: Performance measures comparison of different features and feature selection techniques.

\begin{tabular}{lccc}
\hline Data & $\begin{array}{c}\text { Accuracy (\%) } \\
\text { mean } \pm \text { std }\end{array}$ & $\begin{array}{c}\text { Sensitivity (\%) } \\
\text { mean } \pm \text { std }\end{array}$ & $\begin{array}{c}\text { Specificity (\%) } \\
\text { mean } \pm \text { std }\end{array}$ \\
\hline Full data & $87.35 \pm 0.0878$ & $94.69 \pm 0.1282$ & $86.19 \pm 0.0623$ \\
Pca2 (top2) & $82.32 \pm 0.0055$ & $98.60 \pm 0.0053$ & $24.88 \pm 0.0133$ \\
Pca3 (top3) & $88.00 \pm 0.0071$ & $95.25 \pm 0.0045$ & $67.80 \pm 0.0192$ \\
Pca4 (top4) & $86.47 \pm 0.0673$ & $96.38 \pm 0.0181$ & $72.24 \pm 0.0628$ \\
Pca5 (top5) & $87.33 \pm 0.1244$ & $91.85 \pm 0.0602$ & $90.95 \pm 0.0602$ \\
Pca6 (top6) & $86.69 \pm 0.0692$ & $91.90 \pm 0.1018$ & $91.31 \pm 0.0569$ \\
Wil2 (top2) & $87.78 \pm 0.0032$ & $95.00 \pm 0.0037$ & $64.03 \pm 0.0101$ \\
Wil3 (top3) & $88.00 \pm 0.0055$ & $94.43 \pm 0.0058$ & $77.20 \pm 0.0255$ \\
Wil4 (top4) & $87.56 \pm 0.1673$ & $93.36 \pm 0.2381$ & $74.36 \pm 0.0315$ \\
Wil5 (top5) & $87.56 \pm 0.1482$ & $94.88 \pm 0.2151$ & $93.52 \pm 0.0990$ \\
Wil6 (top6) & $\mathbf{8 8 . 2 0} \pm \mathbf{0 . 1 5 3 1}$ & $\mathbf{9 4 . 9 8} \pm \mathbf{0 . 2 2 0 8}$ & $\mathbf{9 1 . 1 8} \pm \mathbf{0 . 0 9 3 9}$ \\
\hline
\end{tabular}

accuracy. These optimized values are used later by classifier under 3-fold cross-validation over 100 trials in order to get unbiased and stable results.

Table 2 represents all performance measures for different features and feature selection techniques. It includes 3 types of datasets: (i) data with all features, (ii) 5 different sets of high ranked PCA reduced data, and (iii) 5 different sets of high ranked Wilcoxon reduced data. Results reveal that best accuracy is achieved in Wilcoxon data with 6 high ranked features which include eccentricity, Euler number, minor axis length, extent, convex area, and filled area. As false vessels appear in the form of bunches and holes, which exhibit different morphology as compared to true vessels, therefore these best six features best discriminate between true and false vessels.

Tables 3 and 4 illustrate the detailed results for DRIVE and STARE databases, respectively, where all images are evaluated individually and average results are shown in bold fonts.

Table 5 illustrates the comparison made between proposed method and previously proposed methods for DRIVE and STARE datasets on vessel segmentation.

Pictorial results of proposed system for AFIO dataset are shown in Figure 11, where (a) shows colored diseased fundus images, (b) shows vessel maps of images in (a) including both true and false vessels, and (c) shows the final output of the proposed system showing vessel maps of images in (a) without false vessels. Similarly, pictorial results for STARE dataset are shown in Figure 12.

Figures 13 and 14 show STARE and DRIVE results, respectively, which are overlaid over manually segmented results to assess proposed method's performance.

Figure 15 shows pictorial results of Method II: (a) shows images with lesions and then they are inpainted using NBRF algorithm. This smoothes the images by estimating their neighborhood and eventually suppresses the effect of lesions in images as shown in (b). Lastly, blood vessels are segmented from inpainted images which appear with no lesions in final output as depicted in (c).
TABLE 3: Image wise vessel segmentation results for DRIVE database.

\begin{tabular}{lcccccc}
\hline \multirow{2}{*}{ Images } & \multicolumn{3}{c}{ Observer 1 } & \multicolumn{3}{c}{ Observer 2 } \\
& TPR & TNR & ACC & TPR & TNR & ACC \\
\hline 1 & 86.60 & 96.65 & 95.75 & 86.09 & 97.24 & 96.26 \\
2 & 84.49 & 97.65 & 96.30 & 83.07 & 98.35 & 96.81 \\
3 & 79.35 & 96.93 & 95.18 & 83.41 & 97.33 & 96.09 \\
4 & 79.54 & 98.06 & 96.36 & 80.17 & 98.48 & 96.88 \\
5 & 77.91 & 98.19 & 96.29 & 82.87 & 98.52 & 97.25 \\
6 & 79.41 & 97.13 & 95.41 & 79.14 & 97.82 & 96.08 \\
7 & 78.92 & 97.72 & 96.00 & 86.55 & 97.39 & 96.61 \\
8 & 80.71 & 96.26 & 94.92 & 86.30 & 95.95 & 95.31 \\
9 & 82.21 & 97.06 & 95.86 & 79.63 & 97.61 & 96.16 \\
10 & 80.16 & 98.00 & 96.53 & 79.50 & 98.71 & 97.34 \\
11 & 80.25 & 97.11 & 95.60 & 83.67 & 97.63 & 96.47 \\
12 & 84.03 & 97.11 & 95.98 & 85.16 & 97.46 & 96.47 \\
13 & 78.57 & 97.52 & 95.67 & 75.40 & 98.35 & 96.03 \\
14 & 86.63 & 97.18 & 95.74 & 87.78 & 96.95 & 96.26 \\
15 & 86.43 & 96.54 & 96.13 & 82.29 & 97.55 & 96.41 \\
16 & 83.36 & 97.55 & 96.27 & 82.14 & 98.09 & 96.73 \\
17 & 83.19 & 96.13 & 95.04 & 87.43 & 96.52 & 95.85 \\
18 & 86.27 & 96.57 & 95.75 & 82.33 & 98.20 & 96.74 \\
19 & 89.67 & 97.33 & 96.69 & 74.62 & 99.10 & 96.67 \\
20 & 88.23 & 96.77 & 96.14 & 77.36 & 98.30 & 96.36 \\
Average & $\mathbf{8 2 . 8 0}$ & $\mathbf{9 7 . 1 6}$ & $\mathbf{9 6 . 1 4}$ & $\mathbf{8 2 . 2 4}$ & $\mathbf{9 7 . 7 8}$ & $\mathbf{9 6 . 4 4}$ \\
\hline & & & & & &
\end{tabular}

TABLE 4: Image wise vessel segmentation results for STARE database.

\begin{tabular}{lcccccc}
\hline \multirow{2}{*}{ Images } & \multicolumn{3}{c}{ Observer 1 } & \multicolumn{3}{c}{ Observer 2 } \\
& TPR & TNR & ACC & TPR & TNR & ACC \\
\hline 1 & 75.48 & 87.42 & 95.67 & 74.43 & 96.26 & 94.24 \\
2 & 74.26 & 96.70 & 95.20 & 81.47 & 93.94 & 93.25 \\
3 & 88.03 & 96.20 & 95.72 & 79.42 & 92.87 & 92.09 \\
4 & 72.47 & 88.47 & 87.28 & 83.33 & 86.80 & 86.55 \\
5 & 76.32 & 95.97 & 94.19 & 75.83 & 95.00 & 92.99 \\
6 & 88.45 & 96.29 & 95.74 & 74.05 & 96.44 & 93.88 \\
7 & 89.09 & 97.41 & 96.74 & 75.46 & 98.11 & 95.16 \\
8 & 88.51 & 97.38 & 96.72 & 69.52 & 98.51 & 94.53 \\
9 & 88.79 & 97.71 & 97.01 & 74.65 & 97.73 & 94.90 \\
10 & 85.47 & 96.87 & 95.59 & 64.64 & 97.80 & 92.74 \\
11 & 83.84 & 97.46 & 96.49 & 72.36 & 97.93 & 95.00 \\
12 & 86.95 & 97.84 & 97.00 & 79.28 & 98.68 & 96.43 \\
13 & 84.40 & 98.13 & 96.91 & 71.63 & 99.14 & 95.26 \\
14 & 84.55 & 98.25 & 97.01 & 73.30 & 98.92 & 95.52 \\
15 & 81.23 & 97.16 & 95.79 & 77.40 & 98.11 & 95.51 \\
16 & 66.24 & 98.16 & 94.90 & 60.91 & 98.42 & 92.70 \\
17 & 78.72 & 98.64 & 96.85 & 67.92 & 98.91 & 94.85 \\
18 & 81.31 & 98.62 & 97.74 & 80.52 & 98.14 & 97.05 \\
19 & 86.79 & 97.07 & 96.62 & 80.66 & 96.44 & 95.45 \\
20 & 76.60 & 96.08 & 94.78 & 71.41 & 95.38 & 92.99 \\
Average & $\mathbf{8 1 . 8 7}$ & $\mathbf{9 6 . 8 9}$ & $\mathbf{9 5 . 7 2}$ & $\mathbf{7 4 . 4 1}$ & $\mathbf{9 6 . 6 8}$ & $\mathbf{9 4 . 0 5}$ \\
\hline & & & & & &
\end{tabular}


TABLE 5: Comparison of proposed system with existing state of the art on DRIVE and STARE databases.

\begin{tabular}{|c|c|c|c|c|c|c|}
\hline \multirow{2}{*}{ Methods } & \multicolumn{3}{|c|}{ DRIVE } & \multicolumn{3}{|c|}{ STARE } \\
\hline & TPR & TNR & Accuracy & TPR & TNR & Accuracy \\
\hline Emary et al. [11] & 72.1 & 97.1 & 93.9 & 64.9 & 98.2 & 94.7 \\
\hline Aramesh and Faez [25] & 78.4 & 98.26 & 94.8 & - & - & - \\
\hline Imani et al. [26] & 75.82 & 97.48 & 95.25 & 75 & 97.31 & 95.75 \\
\hline Tagore et al. [13] & - & - & 94.24 & - & - & 94.97 \\
\hline Asad et al. [12] & 62.92 & 98.21 & 93.69 & - & - & - \\
\hline Akram and Khan [5] & - & - & 94.69 & - & - & 95.02 \\
\hline Proposed Method (observer 1) & 82.80 & 97.16 & 96.14 & 81.87 & 96.89 & 95.72 \\
\hline Proposed Method (observer 2) & 82.24 & 97.78 & 96.44 & 74.41 & 96.68 & 94.05 \\
\hline
\end{tabular}
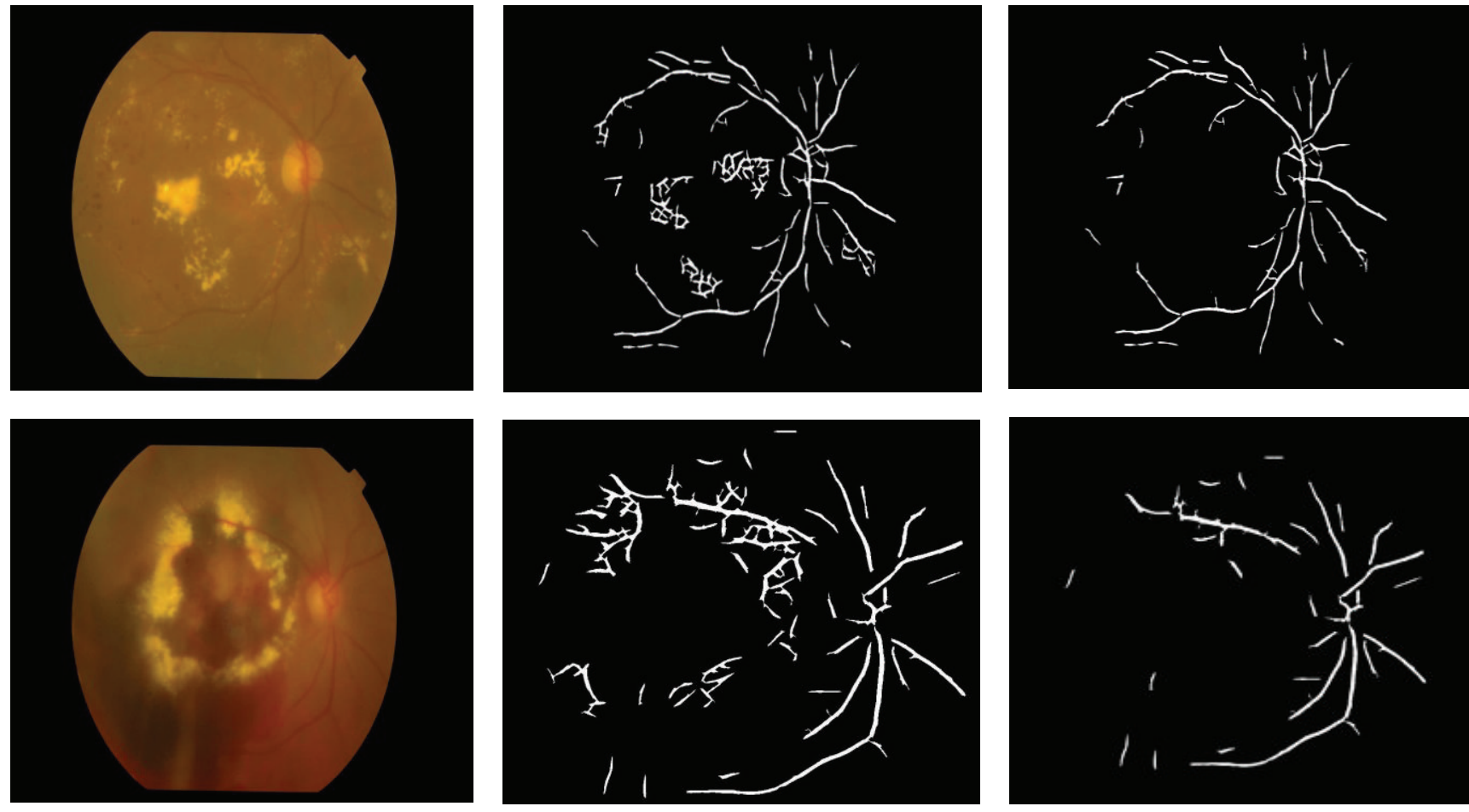

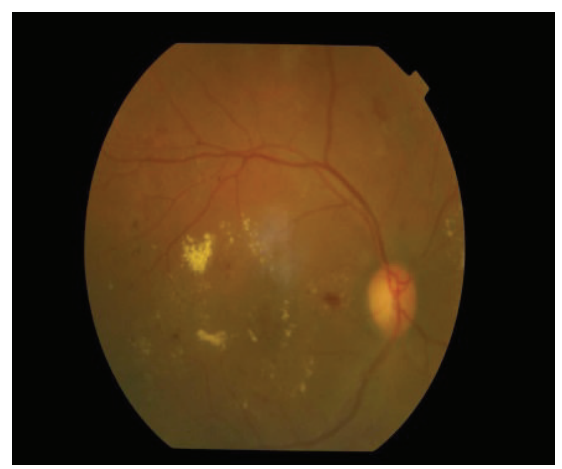

(a)

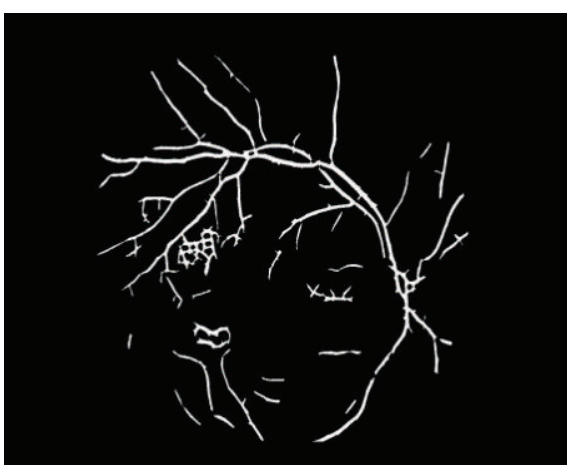

(b)

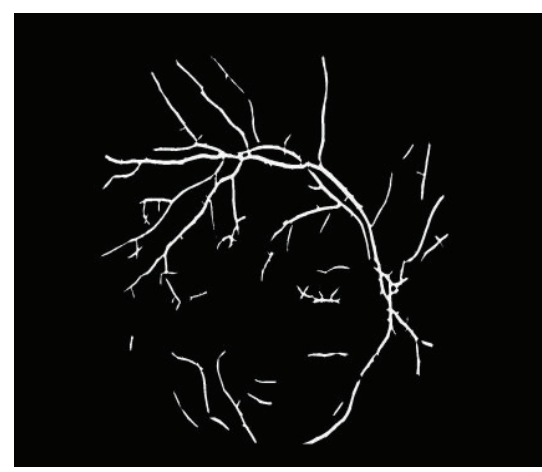

(c)

FIGURE 11: (a) Colored fundus image (from AFIO database). (b) Segmented blood vessels of images in (a). (c) Final output of proposed method (without false vessels). 

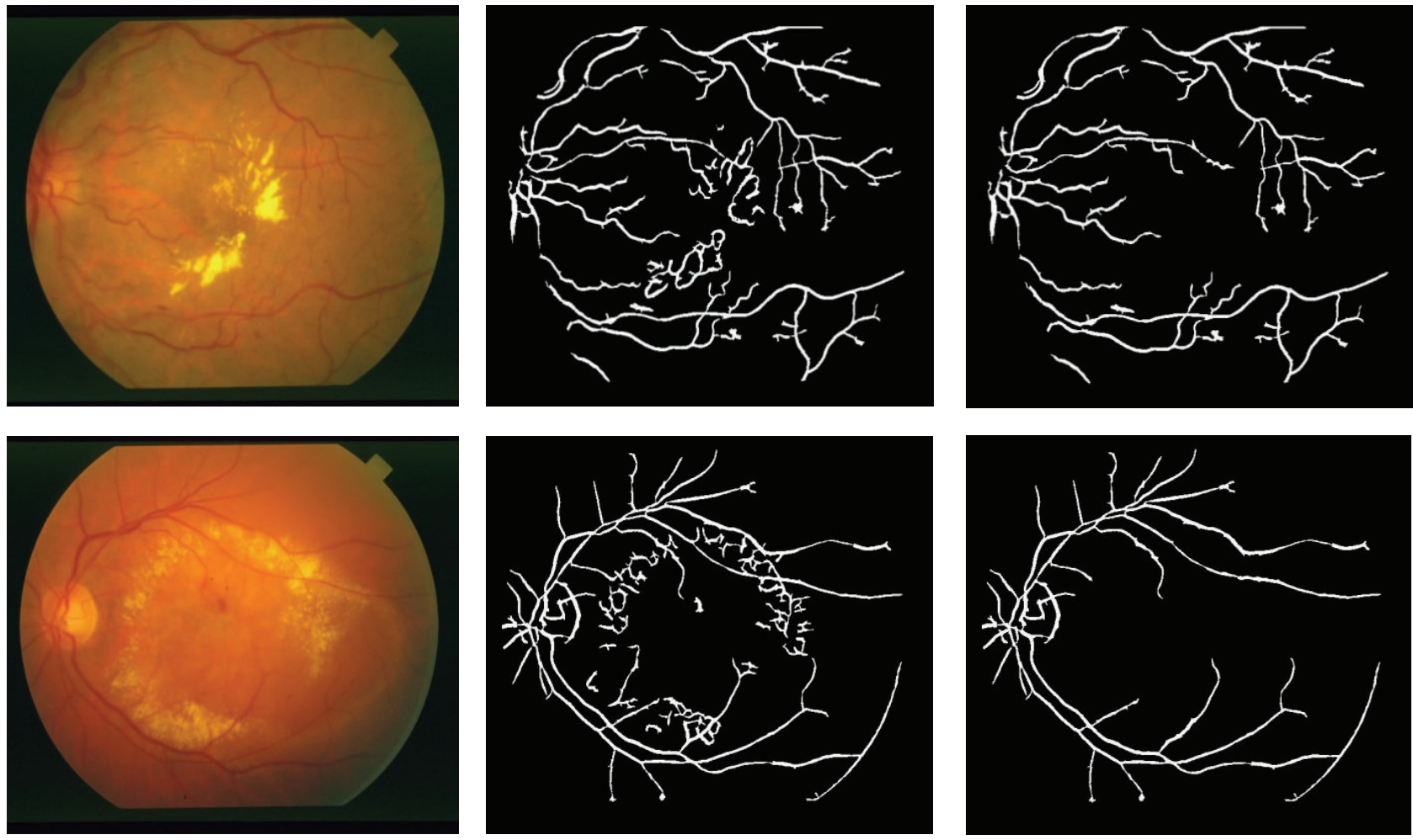

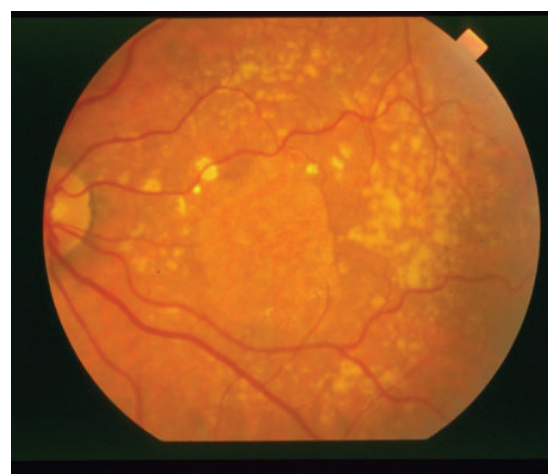

(a)

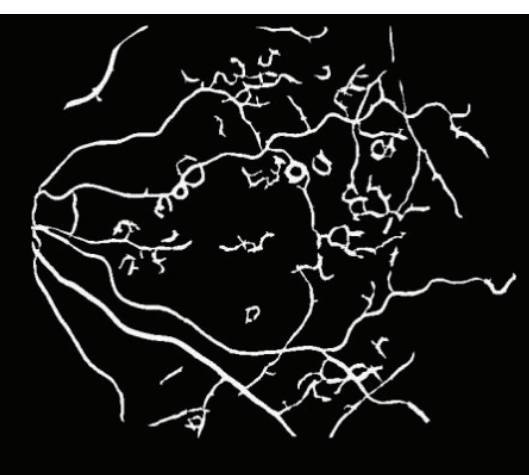

(b)

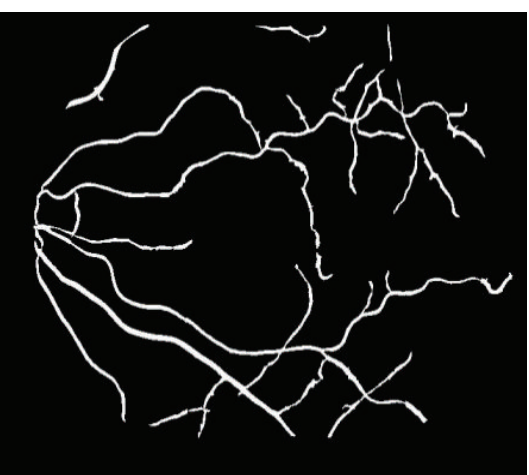

(c)

Figure 12: (a) Colored fundus image (from STARE database). (b) Segmented blood vessels of images in (a). (c) Final output of proposed method (using false vessels).

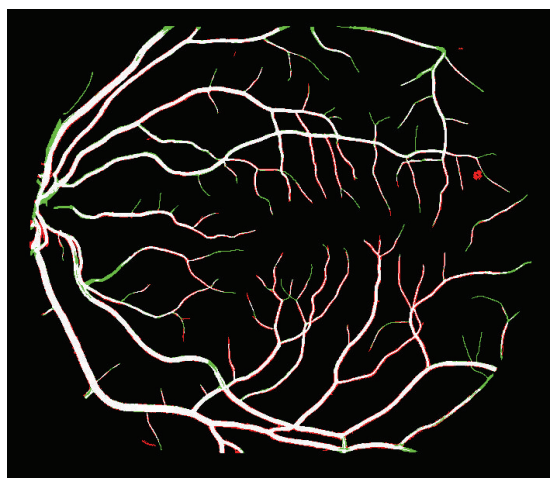

(a)

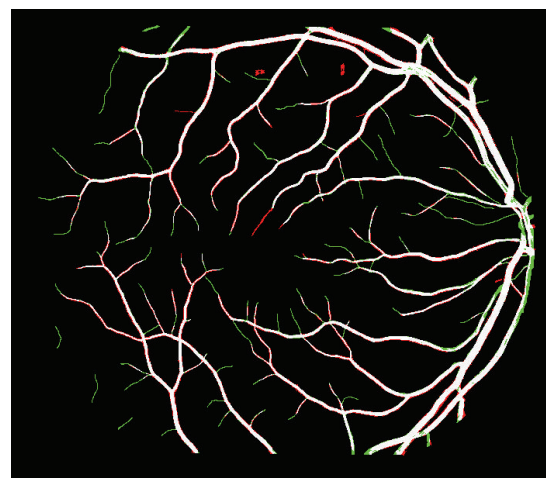

(b)

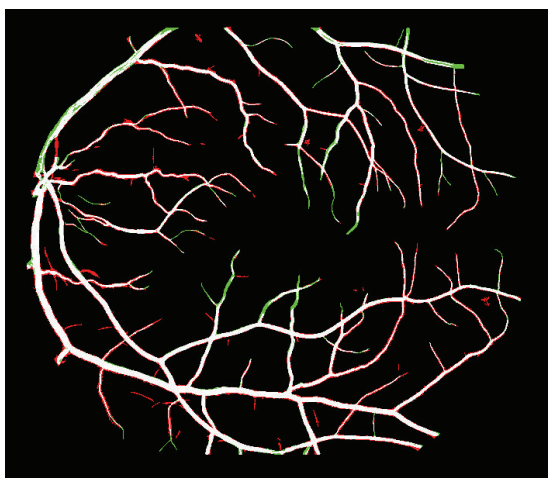

(c)

FIGURE 13: Segmented blood vessels (with STARE) and their comparison with manually marked vessels. White pixels show true positive vessels. Green pixels show false negative vessels. Red pixels show false positive vessels. 


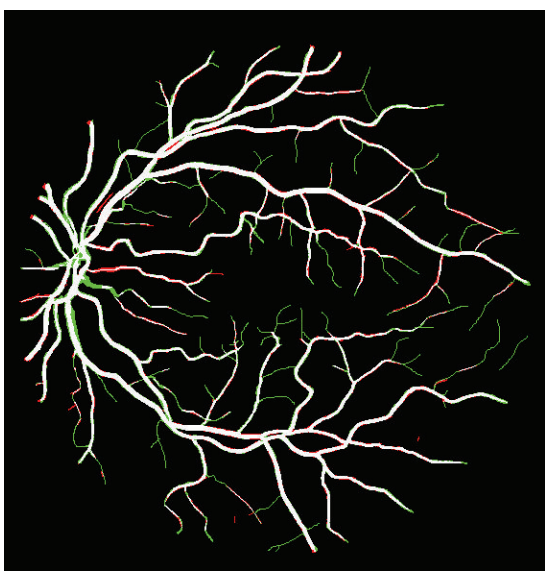

(a)

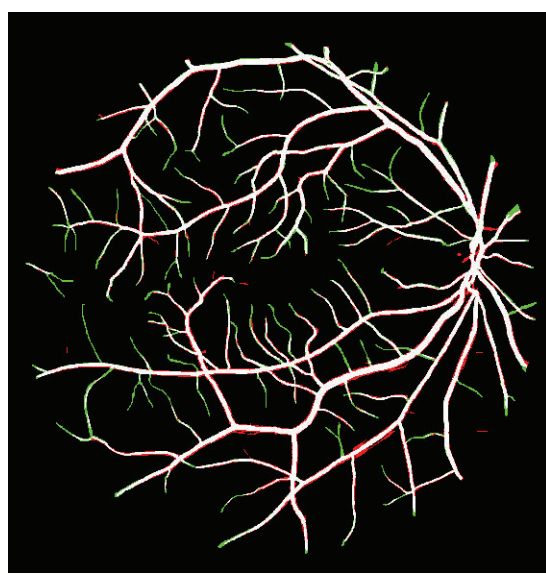

(b)

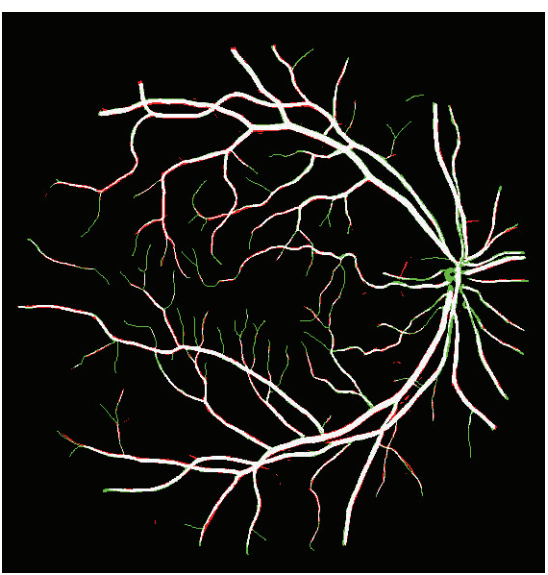

(c)

FIGURE 14: Segmented blood vessels (with DRIVE) and their comparison with manually marked vessels. White pixels show true positive vessels. Green pixels show false negative vessels. Red pixels show false positive vessels.
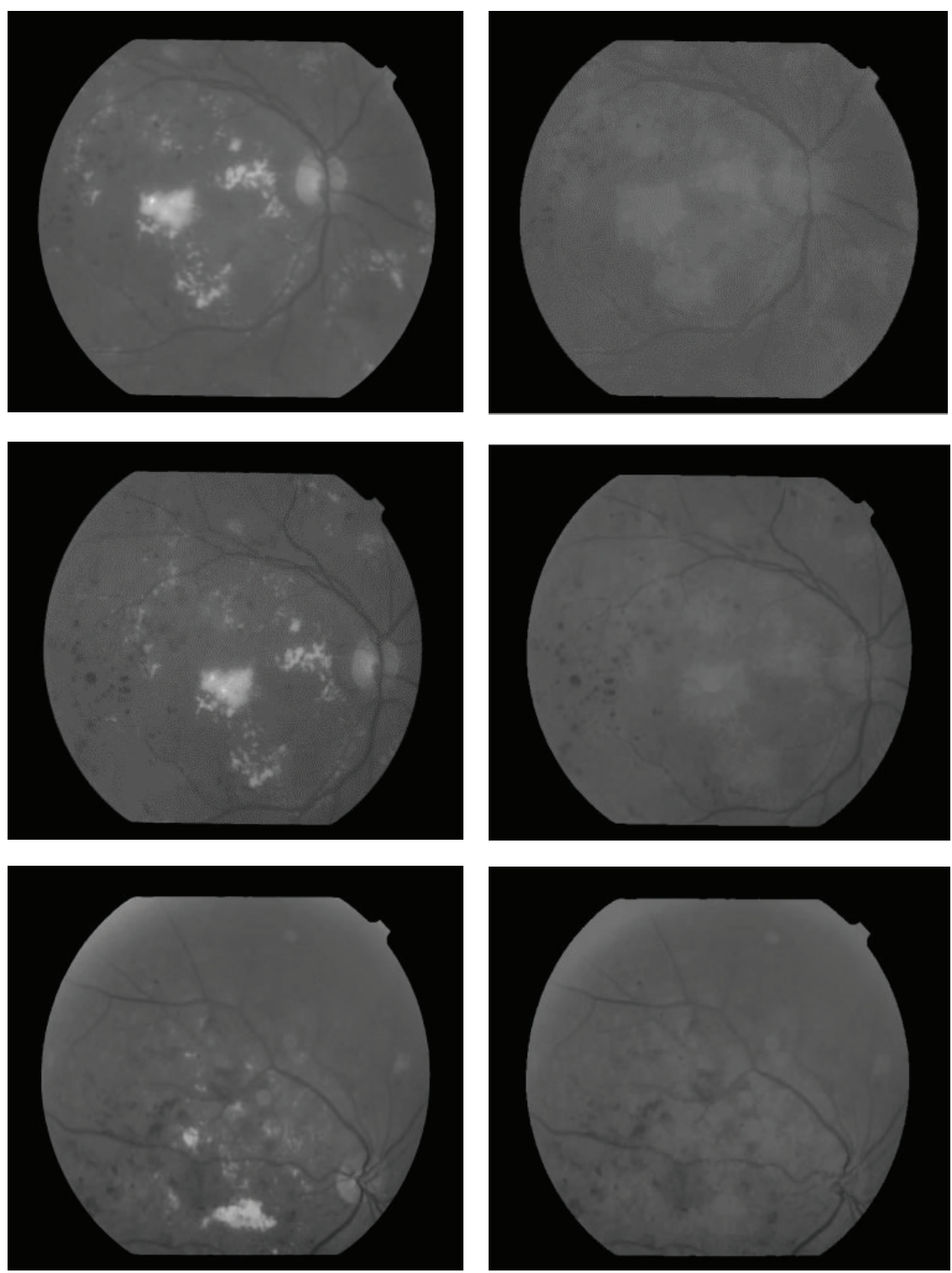

(a)

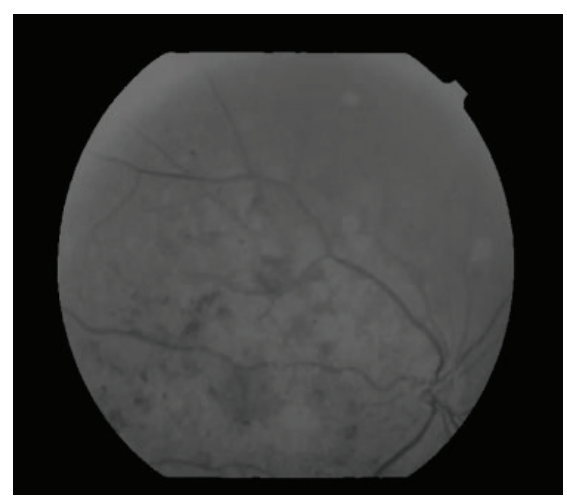

(b)
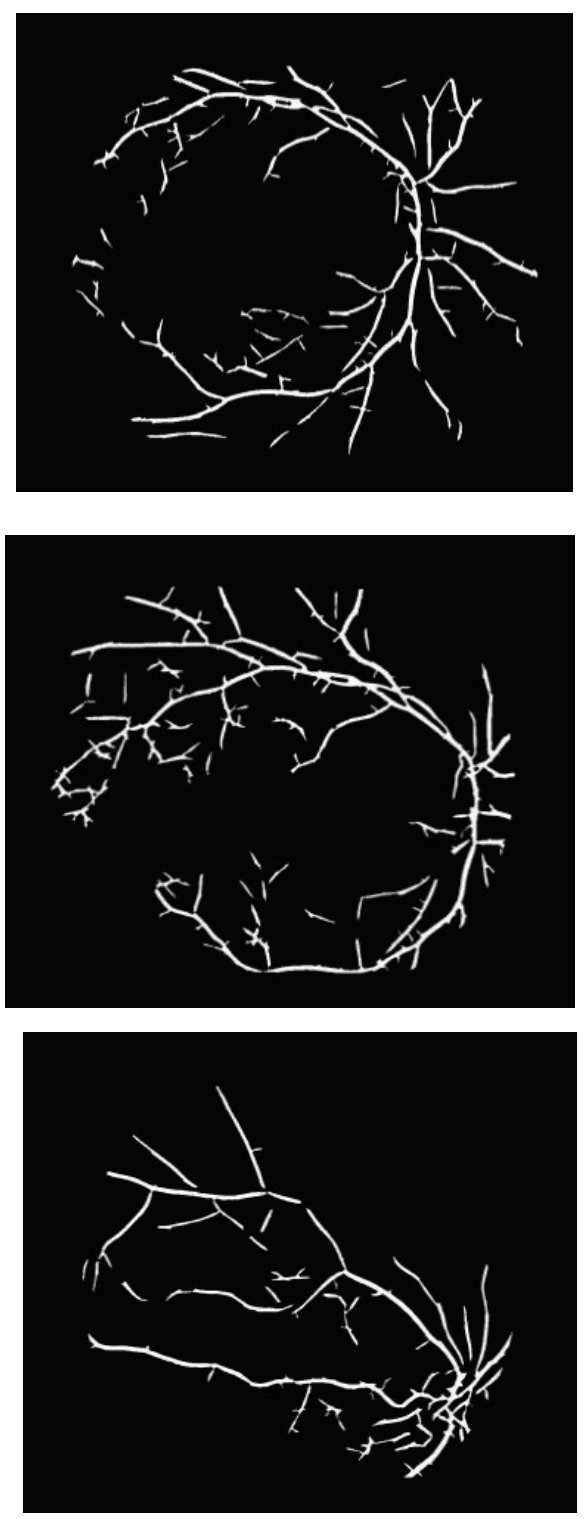

(c)

Figure 15: (a) Green channel images, (b) inpainted results of images in (a), and (c) segmented vessels of images in (b). 

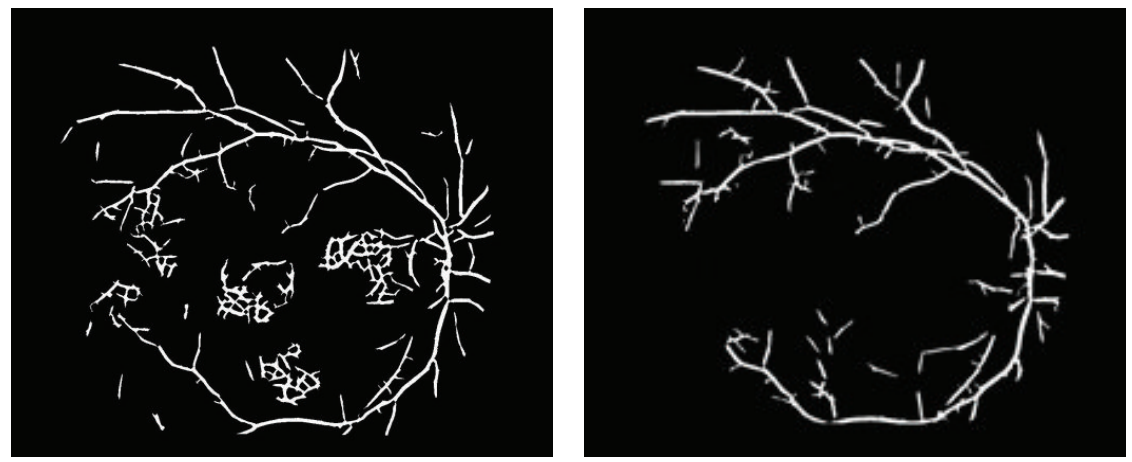

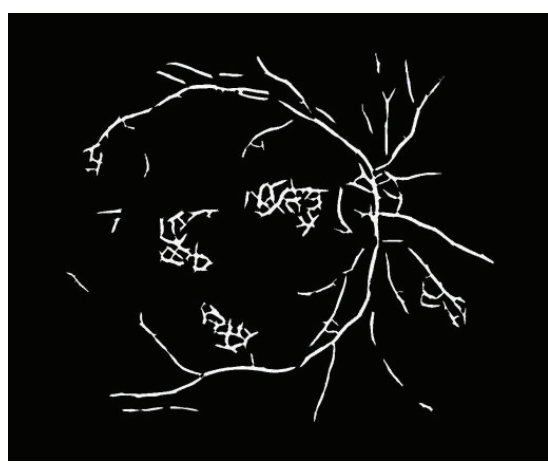

(a)

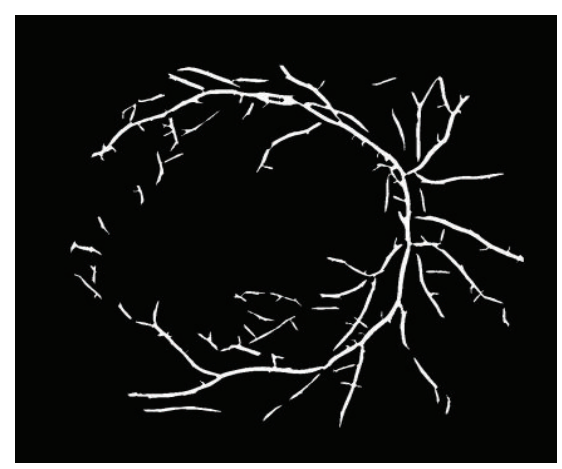

(b)

FIGURE 16: (a) Segmentation results without inpainting. (b) Segmentation results with inpainting.

TABLE 6: Overall accuracy of proposed system using all databases.

\begin{tabular}{lccc}
\hline Database & AFIO (\%) & STARE (\%) & DRIVE (\%) \\
\hline Accuracy & $88.20 \pm 0.1531$ & $95.72 \pm 1.0901$ & $96.44 \pm 0.0050$ \\
\hline
\end{tabular}

Figure 16 shows comparison of segmented images with and without inpainting technique (Method II). It is clearly shown in the results that images appear with false positives which are segmented without applying inpainting technique, while they are significantly removed in images obtained after applying inpainting technique.

Table 6 shows overall accuracy of the system for three AFIO, STARE, and DRIVE databases.

\section{Conclusion}

Blood vessels segmentation is a major step in any computeraided diagnostic system. It has become more challenging in diseased images in the presence of lesions. This paper mainly aims to remove this problem and aims to segment diseased fundus images by removing false vessels due to lesions. It proposes two improved methods for accurate blood vessel segmentation in the presence of lesions. Method I is a supervised method which works on region level and extracts all region based features on the basis of true and false vessels morphology. Then most discriminating features are extracted using feature selection techniques. Lastly, it classifies each region as true or false vessel by using best chosen features. Method II is a classification free method which proposes an algorithm named neighborhood based region filling (NBRF), and it aims to reduce lesions effect by inpainting lesions region before enhancement and segmentation. This is performed by estimating lesions neighborhood. Inpainted images are then enhanced and segmented, which clearly removes false vessels due to lesions. Overall system is tested on DRIVE, STARE, and our own database AFIO. Achieved accuracy of AFIO, STARE, and DRIVE is 88.20.

\section{Conflict of Interests}

The authors declare that there is no conflict of interests regarding the publication of this paper.

\section{Acknowledgments}

This research is funded by National ICT R\&D Fund, Pakistan. The authors are also thankful to Armed Forces Institute of Ophthalmology (AFIO) for their clinical support and help.

\section{References}

[1] M. Usman Akram, S. Khalid, A. Tariq, and M. Younus Javed, "Detection of neovascularization in retinal images using multivariate m-Mediods based classifier," Computerized Medical Imaging and Graphics, vol. 37, no. 5-6, pp. 346-357, 2013.

[2] A. Elbalaoui, M. Boutaounte, H. Faouzi, M. Fakir, and A. Merbouha, "Segmentation and detection of diabetic retinopathy 
exudates," in Proceedings of the International Conference on Multimedia Computing and Systems (ICMCS '14), pp. 171-178, Marrakech, Morocco, April 2014.

[3] D. Sundhara Raja, S. Vasuki, and D. Rajesh Kumar, "Performance analysis of retinal image blood vessel segmentation," Advanced Computing: An International Journal, vol. 5, no. 2/3, pp. 17-23, 2014.

[4] J. Fatima, A. M. Syed, and M. Usman Akram, "A secure personal identification system based on human retina," in Proceedings of the IEEE Symposium on Industrial Electronics and Applications (ISIEA '13), pp. 90-95, Kuching, Malaysia, September 2013.

[5] M. U. Akram and S. A. Khan, "Multilayered thresholding-based blood vessel segmentation for screening of diabetic retinopathy," Engineering with Computers, vol. 29, no. 2, pp. 165-173, 2013.

[6] V. Shanmugam and R. S. D. W. Banu, "Retinal blood vessel segmentation using an extreme learning machine approach," in Proceedings of the 1st IEEE-EMBS Conference on Point-of-Care Healthcare Technologies (PHT '13), pp. 318-321, Bnagalore, India, January 2013.

[7] C. Holbura, M. Gordan, A. Vlaicu, L. Stoian, and D. Capatana, "Retinal vessels segmentation using supervised classifiers decisions fusion," in Proceedings of the IEEE International Conference on Automation Quality and Testing Robotics (AQTR '12), pp. 185-190, Cluj-Napoca, Romania, May 2012.

[8] C. H. Shruthi, N. Ramakrishna, and N. M. Muthukrishnan, "Detection and classification of diabetic retinopathy condition in retinal images," International Journal of Innovative Research in Electronics and Communications, vol. 1, no. 6, 2014.

[9] S. Wang, Y. Yin, G. Cao, B. Wei, Y. Zheng, and G. Yang, "Hierarchical retinal blood vessel segmentation based on feature and ensemble learning," Neurocomputing, vol. 149, pp. 708-717, 2015.

[10] M. M. Fraz, P. Remagnino, A. Hoppe et al., "An ensemble classification-based approach applied to retinal blood vessel segmentation," IEEE Transactions on Biomedical Engineering, vol. 59, no. 9, pp. 2538-2548, 2012.

[11] E. Emary, H. M. Zawbaa, A. E. Hassanien, G. Schaefer, and A. T. Azar, "Retinal blood vessel segmentation using bee colony optimisation and pattern search," in Proceedings of the International Joint Conference on Neural Networks (IJCNN '14), pp. 1001-1006, IEEE, Beijing, China, July 2014.

[12] A. H. Asad, E. Elamry, and A. El Hassanein, "Retinal vessels segmentation based on water flooding model," in Proceedings of the 9th International Computer Engineering Conference (ICENCO '13), December 2013.

[13] M. R. N. Tagore, G. B. Kande, E. V. K. Rao, and B. P. Rao, "Segmentation of retinal vasculature using phase congruency and hierarchical clustering," in Proceedings of the 2nd International Conference on Advances in Computing, Communications and Informatics (ICACCI '13), pp. 361-366, August 2013.

[14] S. Sreng, J.-I. Takada, N. Maneerat et al., "Feature extraction from retinal fundus image for early detection of diabetic retinopathy," in Proceedings of the IEEE Region 10 Humanitarian Technology Conference (R10-HTC '13), pp. 63-66, Sendai, Japan, August 2013.

[15] J. Odstrcilik, R. Kolar, A. Budai et al., "Retinal vessel segmentation by improved matched filtering: evaluation on a new highresolution fundus image database," IET Image Processing, vol. 7, no. 4, pp. 373-383, 2013.

[16] M. D. Saleh and C. Eswaran, "An automated blood vessel extraction algorithm in fundus images," in Proceedings of the IEEE
International Conference on Bioinformatics and Biomedicine (BIBM '12), pp. 482-486, October 2012.

[17] R. Bhattacharjee and M. Chakraborty, "Exudates, retinal and statistical features detection from diabetic retinopathy and normal fundus images: an automated comparative approach," in Proceedings of the National Conference on Computing and Communication Systems (NCCCS '12), pp. 1-6, November 2012.

[18] S. Qamber, Z. Waheed, and M. U. Akram, "Personal identification system based on vascular pattern of human retina," in Proceedings of the Cairo International Biomedical Engineering Conference (CIBEC '12), pp. 64-67, December 2012.

[19] U. M. Akram and S. A. Khan, "Automated detection of dark and bright lesions in retinal images for early detection of diabetic retinopathy," Journal of Medical Systems, vol. 36, no. 5, pp. 31513162, 2012.

[20] C. Liao, S. Li, and Z. Luo, "Gene selection using wilcoxon rank sum test and support vector machine for cancer classification," in Computational Intelligence and Security: International Conference, CIS 2006. Guangzhou, China, November 3-6, 2006, vol. 4456 of Lecture Notes in Computer Science, pp. 57-66, 2007.

[21] Sebastianraschka, 2014, http://sebastianraschka.com/Articles/ 2014_pca_step_by_step.html.

[22] M. U. Akram, A. Tariq, S. A. Khan, and M. Y. Javed, "Automated detection of exudates and macula for grading of diabetic macular edema," Computer Methods and Programs in Biomedicine, vol. 114, no. 2, pp. 141-152, 2014.

[23] J. Staal, M. D. Abràmoff, M. Niemeijer, M. A. Viergever, and B. Van Ginneken, "Ridge-based vessel segmentation in color images of the retina," IEEE Transactions on Medical Imaging, vol. 23, no. 4, pp. 501-509, 2004.

[24] A. Hoover, V. Kouznetsova, and M. Goldbaum, "Locating blood vessels in retinal images by piecewise threshold probing of a matched filter response," IEEE Transactions on Medical Imaging, vol. 19, no. 3, pp. 203-211, 2000.

[25] R. Aramesh and K. Faez, "A new method for segmentation of retinal blood vessels using Morphological image processing technique," International Journal of Advanced Studies in Computer Science and Engineering, vol. 3, no. 1, 2014.

[26] E. Imani, M. Javidi, and H. R. Pourreza, "Improvement of retinal blood vessel detection using morphological component analysis," Computer Methods and Programs in Biomedicine, vol. 118 , no. 3, pp. 263-279, 2015. 

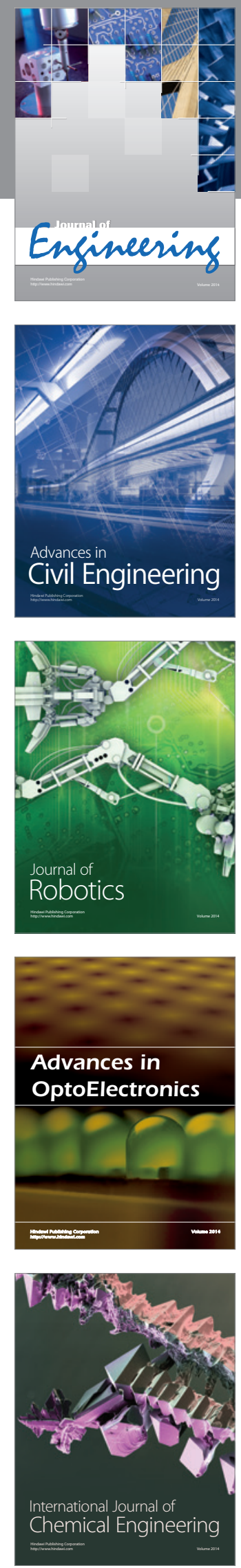

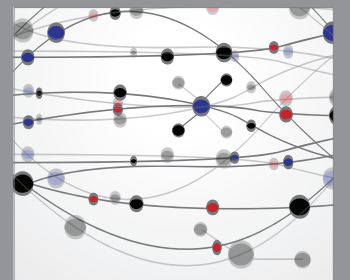

The Scientific World Journal
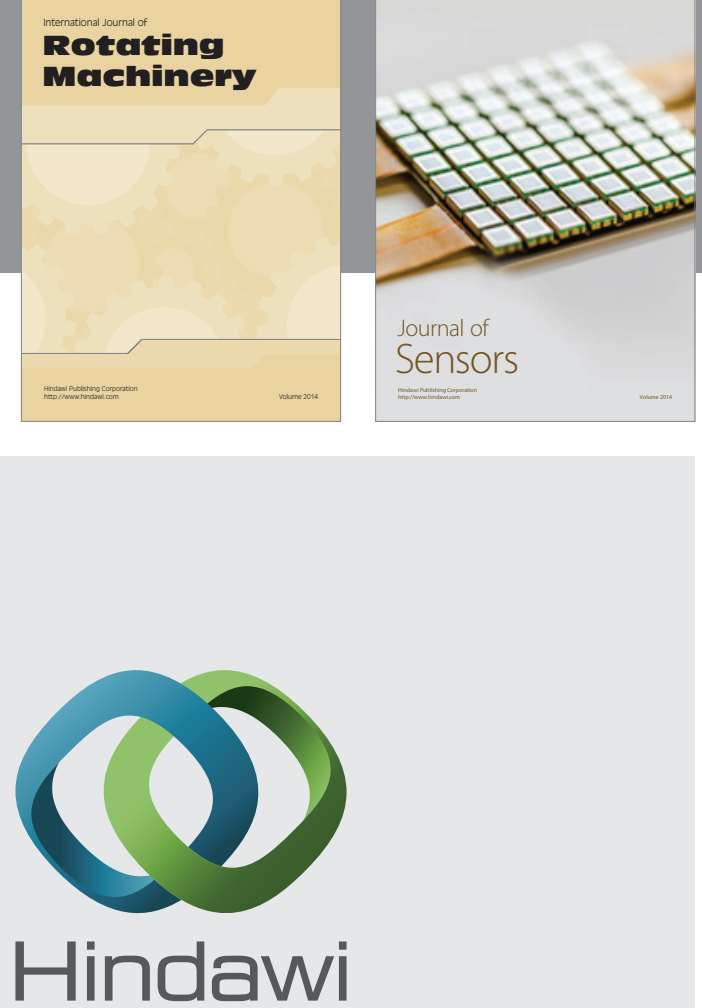

Submit your manuscripts at http://www.hindawi.com
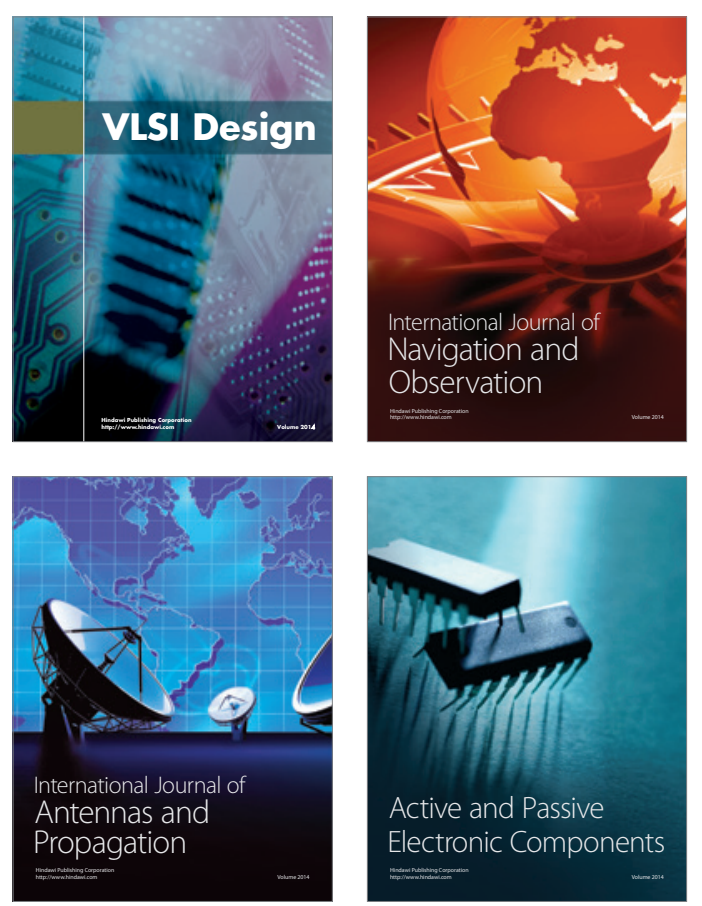
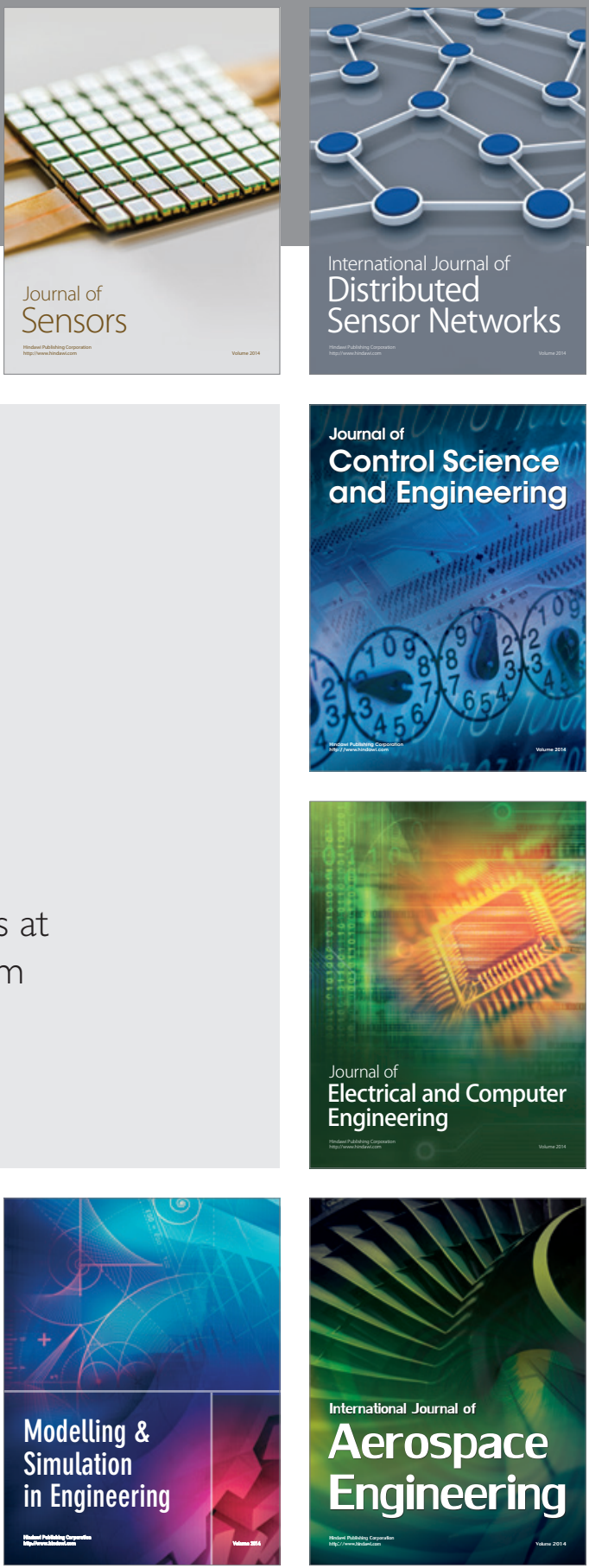

Journal of

Control Science

and Engineering
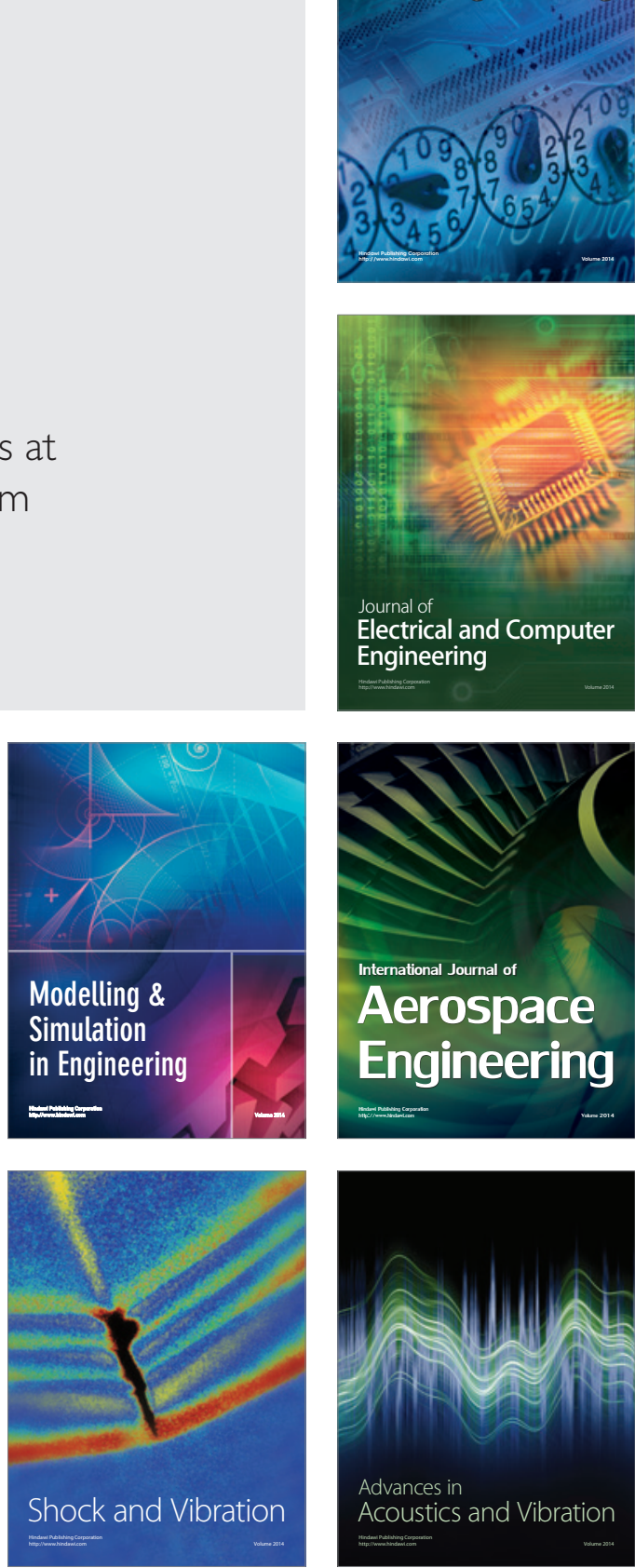